\title{
PESQUISA OPERACIONAL: A COMPARAÇÃO DE DOIS MÉTODOS PARA RESOLUÇÃO DE UM PROBLEMA DE PROGRAMAÇÃO LINEAR
}

\author{
Renato Sousa Botacim ${ }^{1}$, Valderedo Sedano Fontana ${ }^{1}$, Bruno Missi Xavier ${ }^{1}$ e Marcos de \\ Souza ${ }^{1}$
}

\section{RESUMO}

BOTACIM, R.S.; FONTANA, V.S.; XAVIER, B.M.; SOUZA, M. Pesquisa Operacional: a comparação de dois métodos para resolução de um problema de programação linear. Perspectivas Online: Exatas \& Engenharia, v. 09, n. 24, p. 19-33, 2019.

Ante ao crescente desejo pelo lucro, empresas, instituições e pessoas, buscam auxílio em técnicas de Pesquisa Operacional para garantir a maximização de seu capital. Visando atender a essa demanda o mercado, lança diariamente ferramentas e métodos eficientes na resolução dos problemas existentes na Pesquisa Operacional. Desta forma, mediante as inúmeras ferramentas patenteadas e não patenteadas (Open Source) existentes no mercado, serão apresentadas na presente pesquisa conceitualmente duas delas, ambas disponibilizadas gratuitamente.
Posteriormente, foi delimitado um problema/base adaptado, onde foram utilizadas ambos os métodos apresentados na resolução do mesmo problema, com fins comparativos quantos as vantagens e desvantagens, a usabilidade e integridade de cada um. Destarte, diante do exposto será traçada a principal diferença encontrada entre os processos de modelagem, ainda que ambas tenham alcançado o mesmo resultado, evidenciando que ambos apresentam a mesma eficácia na resolução dos métodos.

Palavras-chaves: Pesquisa Operacional, Programação Linear, Simplex, Solver. 


\begin{abstract}
Faced with the growing desire for profit, of charge. Subsequently, an adapted problem / companies, institutions and people, seek help in techniques of Operational Research to base was delimited, where both methods presented in the resolution of the same ensure the maximization of their capital. problem were used, with comparative purposes Aiming to meet this demand the market, launches efficient tools and methods daily in the resolution of the problems in the Operational Research. Thus, through the numerous patented and non-patented tools (Open Source) on the market, two of them will be presented conceptually, both available free as to the advantages and disadvantages, the usability and integrity of each one. Therefore, the main difference found between the modeling processes will be traced, even though both have achieved the same result, evidencing that both have the same effectiveness in the resolution of the methods.
\end{abstract}

Palavras-chaves: Operational Research, Linear Programming, Simplex, Solve

${ }^{1}$ Centro Universitário São Camilo - ES - Rua São Camilo de Léllis, 01, Paraíso, ES, CEP: 29304-910, Brasil.

$\left.{ }^{*}\right)$ e-mail: botacim.renato@gmail.com

Data de recebimento: 25/11/2015. Aceito para publicação: 03/05/2019

Persp. Online: exatas \& eng., Campos dos Goytacazes, 24 (09) 19-33 - 2019 


\section{INTRODUÇÃO}

A Pesquisa Operacional - PO é conceituada como um método científico de leitura de dados para otimização dos no processo de tomada de decisão em diversas áreas do conhecimento. Em linhas gerais, a PO apoia-se em uma abordagem gerencial buscando encontrar a solução com melhor otimização dos dados disponíveis. A origem do estudo do método PO decorre dos acontecimentos da Segunda Guerra Mundial, quando, cientistas norteamericanos se reuniram com o propósito de estudar e resolver problemas de matéria militares.

Segundo Passos (2008) a PO proporciona diversas vertentes, na qual se destaca, a Programação Linear - PL, apresentando-se como uma técnica aplicada a sistemas lineares (equações e inequações) tendo como base modelos previamente estabelecidos. As outras vertentes apresentadas pelo autor, são: Teoria dos jogos; Simulação; Teoria dos Grafos; Teoria das Filas; Programação Dinâmica e a Programação Inteira.

Em uma contextualização histórica, a PO bem como seus métodos, principalmente PL, tiveram sua maior disseminação e popularização aproximadamente durante o fim da segunda Guerra Mundial, por volta 1947, quando o matemático George B. Dantzig criou o denominado método Simplex, que é um algoritmo capaz de viabilizar a solução de muitos problemas matemáticos da programação linear. Vale ressaltar que o essa criação aconteceu enquanto Dantzig trabalhava para a força aérea norte americana (LONGARAY, 2013). Assim, tanto a PO quanto a PL, tem ganhado cada vez mais notoriedade e popularidade nos processos de resolução de problemas.

Desta forma, a difusão do tema e as vantagens proporcionadas pela PO tem influenciado empresas, pessoas e organizações a usufruírem das técnicas de programação linear para a maximização dos lucros e/ou otimização dos gastos. Partindo da premissa anteriormente apresentada pode se extrair a titulo de objeto de analise para a pesquisa $\mathrm{o}$ seguinte problema que simula de maneira fictícia a necessidade de um produtor rural na maximização dos seus lucros no plantio de duas culturas diferentes, emerge a problemática dessa pesquisa: A partir do processo de modelagem para resolução desse problema fictício, por mais simples que ele seja, qual dos métodos, tabular ou simplex, apresenta menor complexidade de execução almejando alcançar com êxito o valor ótimo para as variáveis de decisão buscando a obtenção do lucro máximo?

Visto que a PL é uma das formas mais utilizadas para resolução de problemas pertencentes a área da PO, conforme aponta Silva et al. (2010), a importância do desenvolvimento desta pesquisa, justifica-se quando é observado o real aproveitamento da ferramenta pelos pequenos empreendedores e grandes organizações, estão em crescente busca por maximização dos seus lucros e otimização dos seus gastos, tendo como fundamentação da programação linear e pesquisa operacional no processo de modelagem e resolução de problemas.

Portanto torna-se notório o crescimento da informática, o surgimento e a promoção de ferramentas que auxiliam a resolução dos problemas de programação linear, também cresce, torando o mercado mais competitivo. Visando solucionar um problema de programação linear almejando a maximização ou minimização da função ótima, torna-se uma tarefa menos complexa. Desta forma, o objetivo geral deste trabalho é apresentar métodos capazes de solucionar problemas de PL, além da realização de uma simulação de um problema fictício

Persp. Online: exatas \& eng., Campos dos Goytacazes, 24 (09) 19-33 - 2019 
voltado para a área agrícola, partindo da sua modelagem até a sua solução. Já como objetivos específicos: I) realizar um levantamento bibliográfico minimamente necessário; II) desenvolver a modelagem do problema; III) encontrar a solução ideal para o problema usando dois métodos válidos; IV) comparar qual dos métodos apresenta menor complexidade.

\section{PESQUISA OPERACIONAL}

Conforme o site oficial da Sociedade Brasileira de Pesquisa Operacional (SOBRAPO), a PO é conceituada como uma ciência voltada a resolução de problemas reais de tomada decisão nas mais diversas áreas, procurando alternativas viáveis para alcançar a melhor solução. Ainda segundo a SOBRAPO, a PO originou-se em meio a Segunda Guerra Mundial, devido à complexidade dos problemas militares de logística, tática e estratégia. Deste modo, tanto físicos, quanto matemáticos e engenheiros, transformaram a realidade em modelos matemáticos, assim, conseguiam simular e avaliar os hipotéticos resultados das estratégias analisadas.

Posteriormente a revolução industrial ocorrida no século XIX, notório foi o crescimento da indústria, comercio e organizações de forma geral. Pequenos negócios, com o passar do tempo evoluíram e tornaram-se empreendimentos bilionários. Esta evolução é dada por significativas mudanças, fundamentais, de caráter processual, social e cultural. Desta forma, a aparição de problemas dentro desses empreendimentos é constante, logo, a PO surge para trazer as melhores soluções para os problemas (HILLIER; LIEBERMAN, 2001).

\section{1 Áreas e fases da Pesquisa Operacional}

De acordo com o autor Passos (2008) a PO está empregada em diversas áreas da ciência. Com seu auxílio, pode-se resolver problemas de: locação de recursos e materiais; corte de materiais; transporte, entre muitos outros. Assim, tanto a PL, quanto a PO tem aplicações em diversas áreas como: Carteira de investimentos; Dieta alimentar; Industria petrolífera, moveleira e química; Manufatura; Metalurgia; Agricultura entre inúmeras outras.

Passos (2008) afirma que de modo geral o estudo da PO pode ser distribuído em seis fases, sendo elas: (i) Formulação do problema; (ii) Estudo do problema; (iii) Construção do modelo; (iv) Resolução do modelo; (v) Validação do modelo; (vi) Implementação do modelo.

Já segundo Andrade (2014) para o processo de modelagem não existe um número definido ou pré-estabelecido de fases, pois, podem variar de acordo com a complexidade encontrada em cada problema a ser resolvido. Desta forma, a figura 1, apresenta estas fases na forma de um diagrama.

Persp. Online: exatas \& eng., Campos dos Goytacazes, 24 (09) 19-33 - 2019 


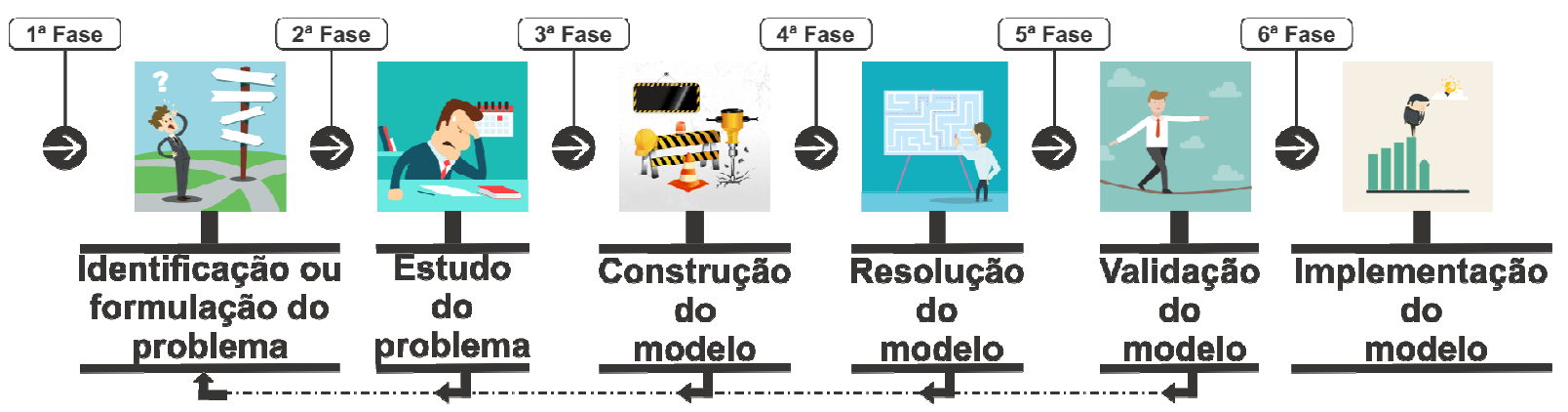

Figura 1: Diagrama das fazes da Pesquisa Operacional. Fonte: Os autores (2015)

\section{PROGRAMAÇÃO LINEAR}

A programação linear, também reconhecida como programação matemática, teve seu início nos meados da década de 40, quando um grupo de cientistas liderado pelo Dr. George B. Dantzig foi recrutado para desenvolver subsídios técnicos para tomadas decisões e organização das tropas nas frentes de batalhas da II Guerra Mundial. Consequentemente em 1947, mediante aos resultados obtidos nas pesquisas o Dr. Dantzig publica o chamado Método Simplex (CAIXETA-FILHO, 2004).

A PL é definida como uma técnica de otimização, que a partir de modelos préestabelecidos, compostos por equações ou inequações, almejam maximizar ou minimizar uma função objeto (função linear), de acordo com suas restrições. Vale ressaltar que os modelos representam a realidade de forma simplificada, logo, o modelo matemático é uma forma de descrever, caracterizar um problema da realidade (PASSOS, 2008).

\subsection{Etapas da resolução da Programação Linear}

Para a resolução de um problema de PL, necessário se faz seguir algumas etapas, que são definidas e conceituadas de acordo com os tópicos descritos a seguir:

a) Construção Modelos: Segundo Silva et al. (2010) a PL é uma das fomas mais utilizada para resolução de problemas em $\mathrm{PO}$, de forma que o uso de técnicas computacionais programáveis facilitam a resolução dos modelos matemáticos que se apresentam de forma concisa a realidade do problema. Desta maneira, os modelos são compostos por uma Função Objetivo (FO), restrições técnicas e variáveis.

b) Variáveis: As variáveis de decisão, também chamadas de variáveis controladas, representam a quantidade de determinados recursos disponíveis na modelagem, como: horas, pessoas, objetos, entre outros, sendo ela o ponto inicial da modelagem. A quantidade desses recursos será a responsável pela otimização máxima ou mínima do problema. Vale ressaltar a importância da definição das variáveis, onde, o sucesso da modelagem são oriundos destas variáveis, pois, a cada iteração realizada, estas assumes diferentes valores até a alcançar a FO e chegar ao resultado ótimo. (PASSOS, 2008).

c) Função Objetivo: Os iniciantes em Pesquisa Operacional, atribuem de maneira errônea, o termo otimização somente para atingir a maior quantidade possível, pois em muitos casos, otimizar também significa estabelecer o melhor valor possível, como em problemas que almejam diminuir os custos da produção de um determinado produto, reduzir o tempo de

Persp. Online: exatas \& eng., Campos dos Goytacazes, 24 (09) 19-33 - 2019 
entrega de um de um produto, entre outros. Desta forma, uma FO de um problema pode buscar a Maximização (Max) ou a Otmização (Min) (LONGARAY, 2013).

d) Restrições: Segundo Longaray (2013) as restrições são consideradas as limitações do problema, compostas por recursos como por exemplo, o tempo disponível de máquinas e/ou pessoas ou a quantidade de matéria prima disponível. Longaray (2013) defende que todos os modelos de otimização são submetidos a restrições, já o Andrade (2014) afirma que na sua maioria as variáveis estão sujeitas as restrições. De qualquer forma, as quantidades de restrições variam de acordo com o problema.

\section{ALGORITMO SIMPLEX}

O Dr. Geórge B. Dantzig em 1947, enquanto trabalhava na força aérea norte americana desenvolvendo soluções para os problemas militares, criou o método Simplex, também conhecido como Algoritmo Simplex, que é capaz de resolver problemas da PL. Seu Funcionamento se dá, através de sucessivas iterações (repetições) desse algoritmo, que busca sempre a maximização ou a otimização de uma FO (PASSOS, 2008). Para Dantzing (1998) o algoritmo Simplex busca por possíveis valores não negativos para um conjunto de variáveis que satisfaçam um sistema de equações lineares. Sua exatidão devem-se às iterações realizadas, pois a cada uma, o algoritmo busca valores que vão de uma extremidade a outra da área de solução, encontrando a melhor solução para o problema em questão.

Conforme Loesch e Hein (2009) o algoritmo Simplex é fundado em três princípios de modo que evitem exaustivas buscas por soluções básicas. À vista disso, o primeiro princípio resume-se em procurar soluções básicas que atendam às restrições. $\mathrm{O}$ segundo, visa a cada iteração melhorar o possível valor para a função objetivo, aumentando em problemas de maximização ou diminuindo em problemas de otimização. Por fim, o terceiro princípio trata dos testes das regras de parada do algoritmo, quando a solução ótima é encontrada ou é ilimitada, ou mesmo se não exista soluções viáveis para o problema.

\subsection{Método Tabular}

Segundo Passos (2008) a solução de problemas de PL utilizando o método Simplex por meio da resolução tabular mostra-se muito eficaz na obtenção dos valores das variáveis de decisão. Este tipo de resolução, apresenta os resultados a cada iteração, sendo possível visualizar qual caminho da zona de solução e o valor que as variáveis estão percorrendo. Por ser resolvido por modelos matemáticos, o método tabular permite solucioná-lo no papel, porém, modelos compostos por muitas variáveis, geram uma grande quantidade no cálculo, então utiliza-se planilhas eletrônicas (Microsoft Excel ou LibreOffice Calc) como auxiliadoras.

O método de resolução do algoritmo Simplex por tableau consiste no processamento do algoritmo em uma base canônica. A cada iteração é gerada uma nova tabela onde está, representa uma solução básica para o problema. Vale ressaltar que o número de iterações para chegar a solução ótima não é constante, visto que a quantidade de variáveis e de restrições mudam de acordo com cada modelagem (LOESCH; HEIN, 2009).

\subsection{Solver}

De acordo Longaray (2013) o Solver é um software aplicado a planilhas eletrônicas

Persp. Online: exatas \& eng., Campos dos Goytacazes, 24 (09) 19-33 - 2019 
que otimiza problemas de Programação Linear utilizando como base o algoritmo Simplex. Foi desenvolvido pela empresa norte americana Frontline Systems, e é disponibilizado como suplemento no Microsoft Excel, linux e Mac, além de estar disponível para download de forma gratuita para estudantes no site oficial de sua desenvolvedora.

A solução de problemas de programação linear por meio do uso de planilhas eletrônicas, depende de uma função objetivo, restrições e uma condição de não-negatividade. Softwares como: Solver, OPM, Lingo e Lindo, são desenvolvidos para conseguirem solucionar os mais diversos e complexos problemas de PL. Entre as inúmeras ferramentas disponíveis no mercado, o Solver é a mais popular, sendo encontrada principalmente em softwares de planilhas eletrônicas como o Microsoft Excel e o LibreOffice Calc (PASSOS, 2008).

\section{METODOLOGIA}

Em acordo com o problema fictício, estruturados com algumas adaptações, de PL proposto neste estudo, tem seu objetivo a maximização dos lucros e está voltado para o segmento agrícola. Para tanto como já estabelecido, dois são os métodos usados para resolução deste problema, sendo primeiro correspondente ao uso da ferramenta Solver, embasado na utilização do software libreoffice Calc na sua versão 4.4.3, ressalta-se que o suplemento Solver se faz presente no Software. O segundo método abordado na resolução do mesmo problema, é o método tabular, tendo como software base o libreoffice Calc, salvaguardando que nessa segunda execução, foi utilizado apenas as funcionalidades da tabelas, logo, sem a utilização do suplemento Solver.

Como base o referencial teórico do presente trabalho, foi utilizado a revisão bibliográfica de artigos científicos disponibilizados pelas bases de busca como Google Acadêmico, bem como livros de autores renomados como: Passos (2008); Loesch e Hein (2009); Caixeta-filho (2004); Silva et al. (2010); Longaray (2013); Andrade (2014) e Prado (2007). Sendo usados ainda os sites oficiais como o da Sociedade Brasileira de Pesquisa Operacional.

Para Gil (2008) natureza da pesquisa se determina como básica e a abordagem do problema define-se como qualitativa. Seus objetivos são caracterizados como exploratórios e ao que se refere aos procedimentos técnicos deste trabalho, são definidos como pesquisas bibliográficas.

\section{RESULTADOS E DISCUSSÃO}

O estudo em questão realizou a solução de problema programação linear adaptado apresentando valores fíctícios. A grande questão é que muitas pessoas podem enfrentam problemas reais similares a esses em seu dia-a-dia. Então cita-se o problema em questão: Um agricultor da cidade de Venda Nova do Imigrante - ES, na expectativa de uma boa colheita, tem à disposição para cultivo em suas terras, dois tipos de grãos: Feijão e Milho. Sua propriedade tem uma área de um Hectare (ha), equivalendo a $10.000 \mathrm{~m} 2$, porém, somente 90 $\%$ das terras são apropriadas para semeadura. Para cada metro quadrado a ser plantado de feijão e milho na propriedade, são necessários os seguintes requisitos como mostra a figura 2.

Persp. Online: exatas \& eng., Campos dos Goytacazes, 24 (09) 19-33 - 2019 


\begin{tabular}{|c|c|c|c|c|}
\hline & $\begin{array}{l}\text { Quilos de } \\
\text { Adubo }\end{array}$ & $\underbrace{}_{\substack{\text { Agua } \\
\text { Litros de }}}$ & $\begin{array}{l}\text { Horas de } \\
\text { Máquina }\end{array}$ & $\begin{array}{ll}\$ & \text { Lucro } \\
\text { em R\$ }\end{array}$ \\
\hline Milho & 0,4 & 3,0 & 0,25 & 1,30 \\
\hline Feijão & 0,8 & 4,0 & 0,5 & 1,70 \\
\hline d & $5.550,00$ & $35.000,00$ & $4.000,00$ & \\
\hline
\end{tabular}

Figura 2: Tabela de restrição e disponibilidade Fonte: Os autores (2015)

Mediante as restrições apresentadas pelo problema, qual seria o melhor valor possível encontrado para as variáveis de decisão, de modo que o produtor chegue ao lucro seu máximo na colheita?

Conforme o problema de PL em questão, o primeiro passo para chegar a sua solução ótima é realização da modelagem dos dados. Segundo Passos (2008) esta modelagem é realizada em cinto etapas, sendo elas: (a) Variáveis de decisão; (b) Função Objetivo; (c) Restrições; (d) Condição de Não-Negatividade; e (e) Modelo. Desta forma, mediante as cinco etapas conceituadas, será realizada a resolução do problema.

Variáveis de decisão: De acordo com o problema, as variáveis de decisão são as que representam a quantidade de cada elemento a ser plantado, assim define-se que:

X1: Quantidade a ser plantada em metros quadrados de feijão

X2: Quantidade a ser plantada em metros quadrados de milho

Função Objetivo: Visto que o objetivo é a maximização do lucro, multiplica-se o lucro de cada produto pela sua quantidade. Conforme apresentado no problema, o valor lucrado com o feijão é de $\mathrm{R} \$ 1,70$ e com o milho $\mathrm{R} \$ 1,30$. Logo:

Max Lucro $=1,70 \mathrm{X} 1+1,30 \mathrm{X} 2$

Restrições: No problema em análise, existem quatro restrições a serem consideradas: uma de área, duas de recursos e uma de mão-de-obra. Desta forma, as restrições podem ser apresentadas da seguinte forma: 
$0,8 \mathrm{X} 1+0,4 \mathrm{X} 2 \leq 5550$ (Restrição da quantidade de Adubo);

$4,0 X 1+3,0 X 2 \leq 35000$ (Restrição da quantidade de Água);

$0,5 \mathrm{X} 1+0,25 \mathrm{X} 2 \leq 4000$ (Restrição da quantidade de Horas de Máquina);

$\mathrm{X} 1+\quad \mathrm{X} 2 \leq 9000$ (Restrição da Áreas disponível para Plantio).

Condição de Não-Negatividade: Esta condição afirma que a quantidade de metros quadrados plantados de feijão ou milho não podem assumir valores negativos:

$\mathrm{X} 1, \mathrm{X} 2 \geq 0$

Modelo: Mediante as etapas anteriores, conforme mostra a tabela 1, a modelagem final do problema apresenta-se da seguinte forma:

Tabela 1: Modelagem completa do problema. Fonte: Os autores (2015)

\begin{tabular}{|c|c|c|c|c|c|c|}
\hline Função Objetivo & $\operatorname{Max} L=1,7$ & $\mathrm{X} 1+$ & 1,3 & $\mathrm{X} 2$ & & \\
\hline \multirow{5}{*}{ Sujeito à: } & 0,8 & $\mathrm{X} 1+$ & 0,4 & $\mathrm{X} 2$ & $\leq$ & 5550 \\
\hline & 4 & $\mathrm{X} 1+$ & 3 & $\mathrm{X} 2$ & $\leq$ & 35000 \\
\hline & 0,5 & $\mathrm{X} 1+$ & 0,25 & $\mathrm{X} 2$ & $\leq$ & 4000 \\
\hline & & $\mathrm{X} 1+$ & & $\mathrm{X} 2$ & $\leq$ & 9000 \\
\hline & & $\mathrm{X} 1, \mathrm{X} 2$ & & & & 0 \\
\hline
\end{tabular}

Após o término da modelagem, o próximo passo é achar o valor ideal para as variáveis de decisão. Assim, uma das formas utilizadas para a solução do modelo é através de planilhas eletrônicas, especificamente, utilizando o suplemento Solver, que é disponibilizado de forma gratuita no software LibreOffice Calc.

Para a resolução do problema através do Solver, é necessário seguir algumas etapas. Nestas etapas, são passados os parâmetros de forma que o Solver consiga interpretar e, consequentemente, resolver o problema. A figura 3, apresenta de forma geral, a área de trabalho do LibreOffice Calc, já resolvido e com todas as etapas prontas. 


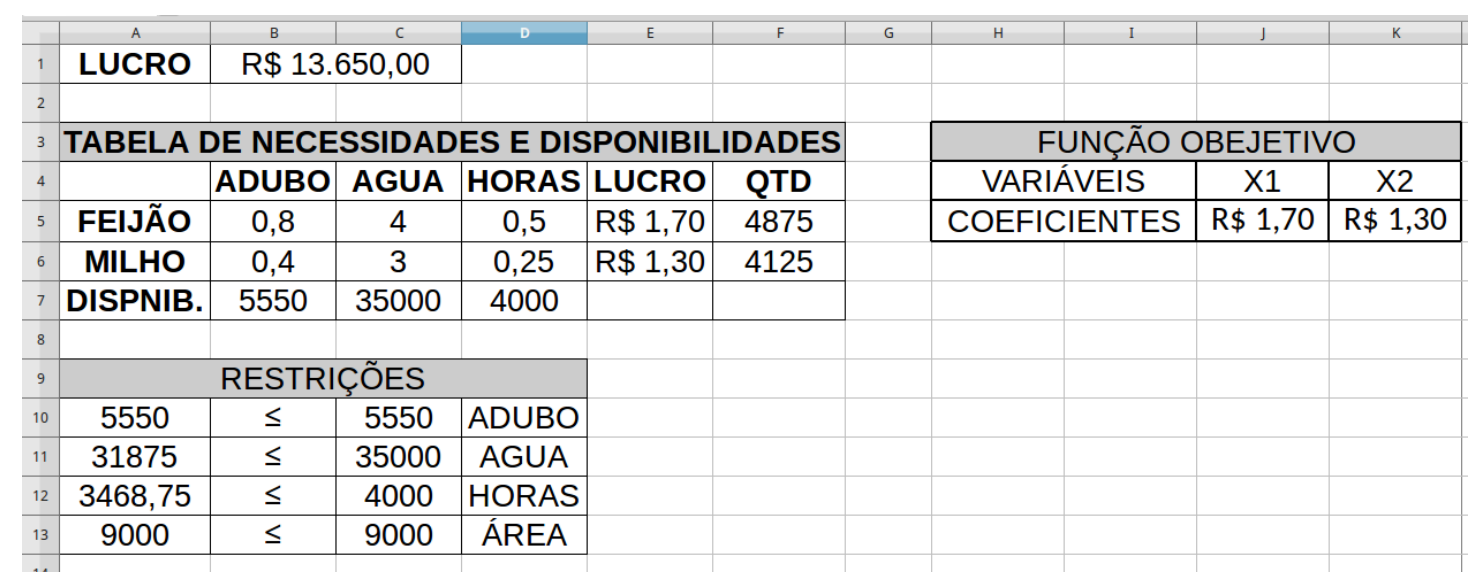

Figura 3: Área de trabalho do LibreOffice Calc preenchida Fonte: Os autores (2015)

Seguindo uma ordem cronológica das etapas a serem realizadas, a primeira, é a inserção da função objetivo na área de trabalho do LibreOffice Calc, sendo representada nas células (H3 a K5) conforme mostra a figura 3.

A segunda etapa é a inserção das restrições. Para facilitar o entendimento e a resolução do problema, é elaborada uma tabela com todas as variáveis e suas necessidades e disponibilidades, conforme mostrado nas células (A3 até F7) da figura 3. Também é reservado uma célula para o valor de cada variável de decisão, célula (F5) para o feijão e célula (F6) para o milho. $\mathrm{O}$ posicionamento das células, podem variar de acordo com quem resolve o problema.

A partir da elaboração da tabela com as variáveis, necessidades e disponibilidades, vide células (A3 até F7) da figura 3, a criação das funções no LibreOffice Calc referente a parametrização das restrições torna-se mais fácil. Assim, as restrições do problema são distribuídas na tabela de restrições como mostra as células (A9 até D13).

Cada linha da tabela de restrições apresentada na figura 3, representa uma restrição do problema. A primeira coluna é composta pela função =SOMARPRODUTO. Esta função faz a soma da multiplicação dos valores das restrições pelas variáveis de decisão, como um exemplo para essa função: "=SOMARPRODUTO(C5:C6;F5:F6)", onde C5 E C6 representa a quantidade de água gasta pelo feijão e milho, respectivamente. Já as células F5 E F6, representam as variáveis de decisão. A segunda coluna contém um símbolo que representa que todas as restrições devem ser menores ou iguais ao seu valor total disponível, representado na terceira coluna. Já a última, para facilitar o entendimento, exibe o nome da restrição.

O último passo é definir a célula que receberá a representação do valor da função objetivo. A célula determinada, será composta pela função "=SOMARPRODUTO", porém, por se tratar de um problema de maximização, esta função somará o produto das variáveis de decisão pelo lucro das mesmas. Conforme mostra as células (A1 a C1) da figura 3.

Para concluir o processo de resolução, uma vez que todas as etapas já estejam finalizadas, basta abrir o suplemento solver e realizar a parametrização das informações necessárias. Assim, na janela de parametrização, na opção "Célula Objetivo" será informada a

Persp. Online: exatas \& eng., Campos dos Goytacazes, 24 (09) 19-33 - 2019 
célula (\$B\$1) referente ao lucro. Nas opções de: "Otimizar para" é marcada a opção "Máximo". No campo de "Células Variáveis", será indicada as células (\$F\$5:\$F\$6) referente as variáveis de decisão. Por fim, é definido o conjunto de restrições, serão indicadas cada restrição do problema. Após todos os campos preenchidos, é só clicar no botão resolver. A figura 4, apresenta a tela do suplemento Solver preenchida com a parametrização dos valores necessários.

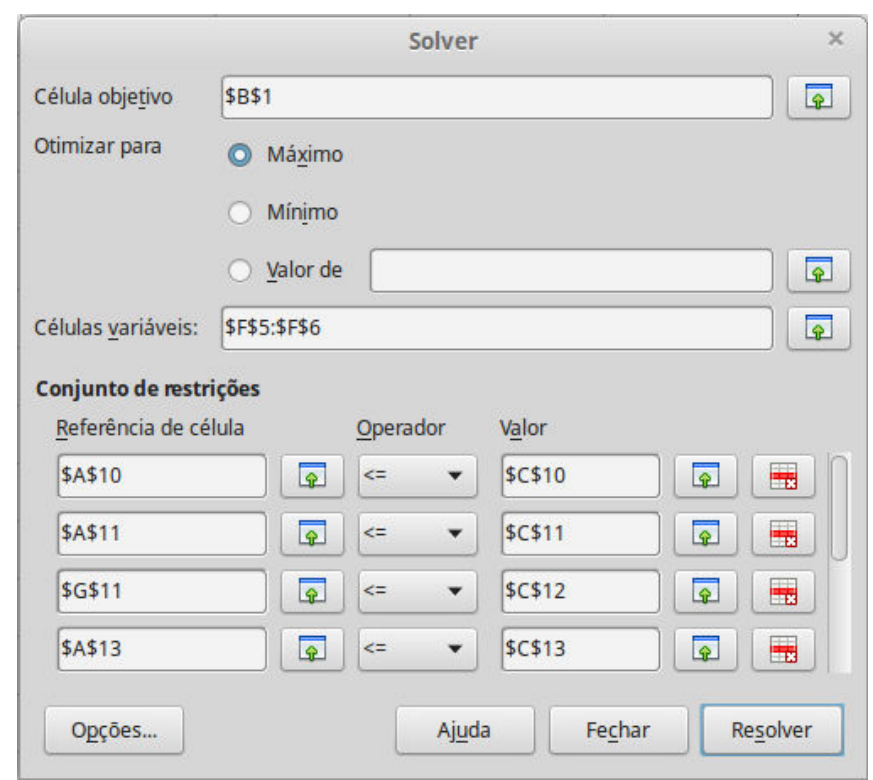

Figura 4: Tela do suplemento Solver Fonte: Os autores (2015)

Note-se que após a mensagem de conclusão, as células referentes ao lucro e variáveis de decisão receberam valores. Os valores assumidos pelas células são os melhores possíveis, nenhum outro valor que inserido manualmente, resultará em um maior lucro sem que nenhuma restrição seja excedida. Desta forma, os resultados obtidos foram os seguintes: X1 ou Quantidade de metros quadrados plantados de feijão igual a 4875; X2 ou Quantidade de metros quadrados plantados de milho igual a 4125 e o Lucro Máximo foi igual a $\mathrm{R} \$$ 13650,00 .

Observe que nem todas as variáveis chegaram ao seu limite, a quantidade de água e de horas de máquina sobraram, enquanto a quantidade de adubo e da área alcançaram seu limite. Isso indica que, se o produtor tivesse uma área de plantio maior e, uma quantidade maior de adubo, seu lucro também aumentaria, entendendo que sobraram outros recursos necessários.

A segunda forma de resolução desse problema de PL, é através do uso do método tabular. Ao contrário do Solver, este método depende apenas de funções matemáticas, sendo possível resolvê-lo por planilhas eletrônicas ou manualmente no papel. Todavia, para a resolução também será utilizado LibreOffice Calc.

Na resolução do problema pelo método tabular, primeiro passo é converter o modelo, de forma canônica (inequações) para a forma padrão (equações). Vale ressaltar que todas as inequações que forem menores ou iguais $(\leq)$ serão acrescidos de uma folga e todas que forem

Persp. Online: exatas \& eng., Campos dos Goytacazes, 24 (09) 19-33 - 2019 
maior ou igual $(\geq)$ serão decrescidas de uma folga. A tabela 2 mostra a conversão completa.

Tabela 2 - Conversão da forma canônica para padrão. Fonte: Os autores (2015)

\begin{tabular}{|c|c|c|c|c|c|c|c|c|c|c|c|c|c|c|}
\hline \multicolumn{7}{|c|}{ Forma Canônica } & \multicolumn{8}{|c|}{ Forma Padrão } \\
\hline \multirow[t]{6}{*}{$\operatorname{Max} L=$} & 1,7 & $\mathrm{X} 1$ & +1 & 1,3 & $\mathrm{X} 2$ & & $\operatorname{Max} \mathrm{L}=$ & $-1,7$ & X1 - & 1,3 & X2 & & & $=$ \\
\hline & 0,8 & $\mathrm{X} 1$ & +0 & 0,4 & $\mathrm{X} 2$ & $\leq 5550$ & & 0,8 & $\mathrm{X} 1+$ & 0,4 & $\mathrm{X} 2+$ & & & $=5550$ \\
\hline & 4 & $\mathrm{X} 1$ & + & 3 & $\mathrm{X} 2$ & $\leq 35000$ & & 4 & $\mathrm{X} 1+$ & 3 & X2 & $+F 2$ & & $=35000$ \\
\hline & 0,5 & $\mathrm{X} 1$ & & 0,25 & $\mathrm{X} 2$ & $\leq 4000$ & & 0,5 & $\mathrm{X} 1+$ & 0,25 & X2 & & $F 3$ & $=4000$ \\
\hline & & $\mathrm{X} 1$ & & & $\mathrm{X} 2$ & $\leq 9000$ & & & $\mathrm{X} 1+$ & & X2 & & $+F 4$ & $=9000$ \\
\hline & & $\mathrm{X} 1,2$ & & & & $\geq$ & & & $\mathrm{X} 1, \mathrm{X} 2$ & $F 1, F$ & $2, \mathrm{~F} 3$ & & & $\geq$ \\
\hline
\end{tabular}

A partir da conversão, é possível construir a tabela com a situação inicial, conforme mostra a figura 5 .

\begin{tabular}{c|c|c|c|c|c|c|c|c|c|}
\hline & A & B & C & D & E & F & G & H & I \\
\hline 1 & BASE & X1 & X2 & F1 & F2 & F3 & F4 & C & SAI \\
\hline 2 & F1 & 0,8 & 0,4 & 1 & 0 & 0 & 0 & 5550 & 6937,5 \\
\hline 3 & F2 & 4 & 3 & 0 & 1 & 0 & 0 & 35000 & 8750 \\
\hline${ }_{4}$ & F3 & 0,5 & 0,25 & 0 & 0 & 1 & 0 & 4000 & 8000 \\
\hline${ }_{5}$ & F4 & 1 & 1 & 0 & 0 & 0 & 1 & 9000 & 9000 \\
\hline 6 & Z & $-1,7$ & $-1,3$ & 0 & 0 & 0 & 0 & 0 & 0 \\
\hline
\end{tabular}

Figura 5: Tabela inicial do método tabular Fonte: Os autores (2015)

Os preenchimentos das células são decorrentes dos valores encontrados na conversão realizada anteriormente. Para iniciarmos a resolução, devemos saber qual será a coluna pivô (coluna que possui a maior contribuição negativa), bom base em nossa tabela, a variável X1 (coluna B). A definição da linha que irá sair é o menor valor positivo diferente de zero, resultante do quociente do valor da constante representado por $\mathrm{C}$ (coluna $\mathrm{H}$ ) pela valor da coluna pivô (coluna B). Desta forma, a linha que sai refere-se a folga um (linha 2). Após a definição de quem sai, é realizada a primeira iteração do método. É mantida a mesma estrutura da tabela, porém, na linha dois referente a folga um, será colocada X1, pois, foi a coluna pivô do quadro inicial.

Os valores das células internas, são definido por três etapas, onde a primeira etapa é para descobrir o valor da nova linha que entrou, dividindo cada célula que saio pela célula pivô. A segunda etapa é descobrir o valor das linhas que sobraram, onde cada célula que entrou é multiplicado pelo pivô negativado da célula que sobrou, somado com todas células que sobraram. Já a terceira etapa é descobrir o valor do $Z$, assim, é multiplicado o valor de cada célula que entrou pelo pivô negativado da célula $Z$ somado com todas células de $Z$ que sobraram. Ressalta-se que estes passos valem para toda e qualquer iteração, assim, a figura 6 apresenta os valores da primeira iteração. Para melhor compreensão, na frente da tabela, foi inserido um quadro informativo com as células e sua respectivas funções. 


\begin{tabular}{|c|c|c|c|c|c|c|c|c|c|c|c|}
\hline & A & B & c & D & $E$ & $\mathrm{~F}$ & G & H & I & K & $\mathrm{L}$ \\
\hline 1 & BASE & $\mathrm{X} 1$ & $X 2$ & F1 & $\mathrm{F} 2$ & F3 & F4 & C & SAI & & \\
\hline 2 & F1 & 0,8 & 0,4 & 1 & 0 & 0 & 0 & 5550 & 6937,5 & & \\
\hline 3 & $\mathrm{~F} 2$ & 4 & 3 & 0 & 1 & 0 & 0 & 35000 & 8750 & & \\
\hline 4 & F3 & 0,5 & 0,25 & 0 & 0 & 1 & 0 & 4000 & 8000 & & \\
\hline 5 & F4 & 1 & 1 & 0 & 0 & 0 & 1 & 9000 & 9000 & & \\
\hline 6 & Z & $-1,7$ & $-1,3$ & 0 & 0 & 0 & 0 & 0 & 0 & & \\
\hline \multicolumn{12}{|l|}{7} \\
\hline 8 & BASE & $\mathrm{X} 1$ & $\mathrm{X} 2$ & F1 & $\mathrm{F} 2$ & F3 & F4 & C & SAI & CÉLULA & FUNÇÃO \\
\hline 9 & $\mathrm{X} 1$ & 1 & 0,5 & 1,25 & 0 & 0 & 0 & 6937,5 & 13875 & B9 & $=\mathrm{B} 2 / \$ \mathrm{~B} \$ 2$ \\
\hline 10 & $\mathrm{~F} 2$ & 0 & 1 & -5 & 1 & 0 & 0 & 7250 & 7250 & B10 & $=\mathrm{B} 9 *(-\$ \mathrm{~B} \$ 3)+\mathrm{B} 3$ \\
\hline 11 & F3 & 0 & 0 & $-0,63$ & 0 & 1 & 0 & 531,25 & 0 & B11 & $=\mathrm{B} 9 *(-\$ B \$ 4)+\mathrm{B} 4$ \\
\hline 12 & F4 & 0 & 0,5 & $-1,25$ & 0 & 0 & 1 & 2062,5 & 4125 & B12 & $=\mathrm{B} 9 *(-\$ B \$ 5)+\mathrm{B} 5$ \\
\hline 13 & Z & 0 & $-0,45$ & 2,125 & 0 & 0 & 0 & 11793,75 & $-26208,3$ & B13 & $=\mathrm{B} 9 *(-\$ B \$ 6)+\mathrm{B} 6$ \\
\hline 14 & & & & & & & & & & & \\
\hline
\end{tabular}

Figura 6: Resultado de entrada dos valores após a primeira iteração Fonte: Os autores (2015)

O símbolo "\$” referência absoluta é utilizado nas funções para travar a célula, que no caso é a coluna pivô. Desta forma, quando arrastamos a função para as demais células, a coluna pivô, não se altera.

A quantidade de iterações irá variar de acordo com cada problema. Elas devem acontecer enquanto houver valores negativos na linha $Z$. No problema modelado, note que ainda há valores negativos na linha Z, especificamente na célula C13. Desta forma, será necessário a realização de mais uma iteração.

Após realizar todas as etapas da segunda iteração, perceba que na linha Z (linha 20) não há mais nenhum valor negativo, isso indica que não será mais necessário uma iteração. Logo, a figura 7, mostra o último quadro completo já com o resultado final do problema.

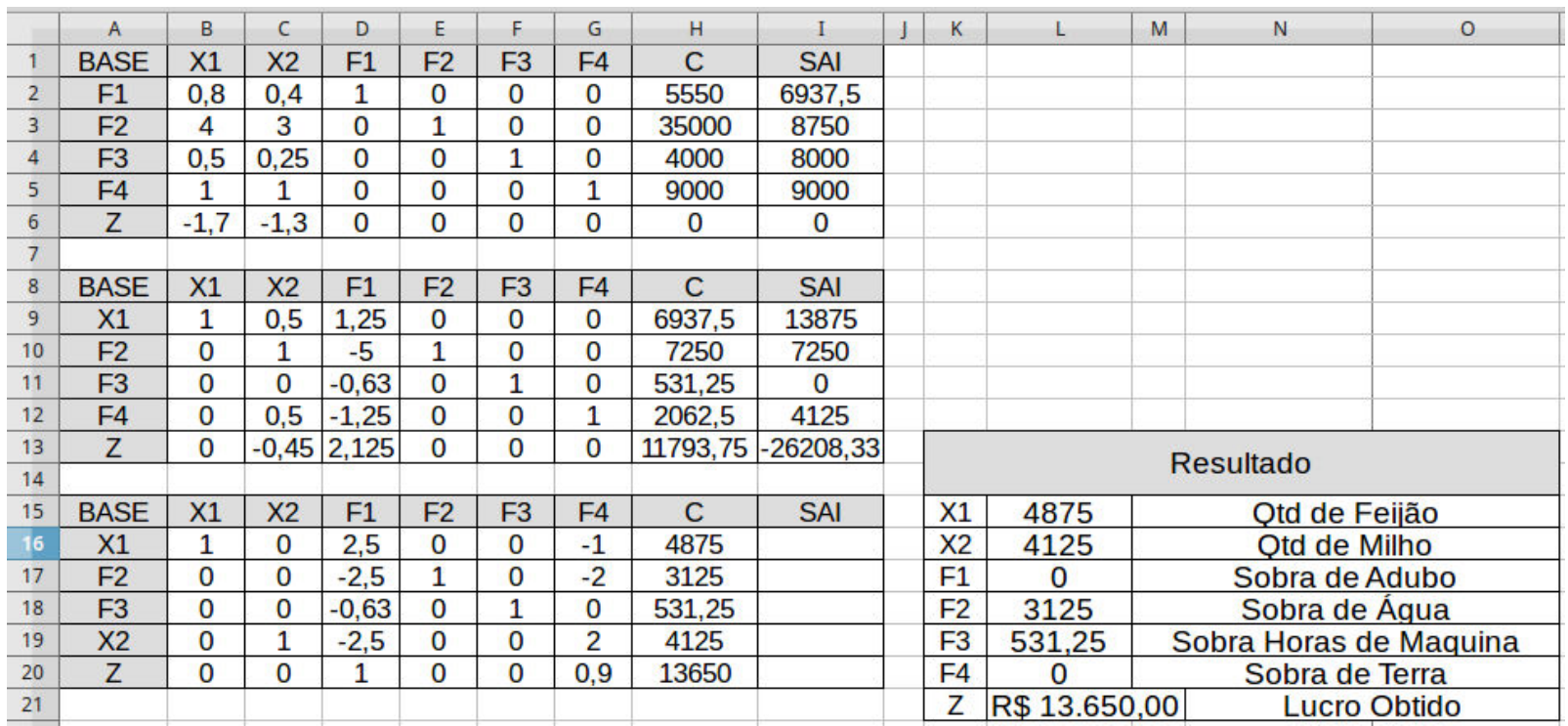

Figura 7: Resultado de entrada dos valores após a primeira iteração Fonte: Os autores (2015)

Visto que somente quem está na base tem valor, a folga 1 e 4 não permaneceram na base, indicando que foi utilizado toda a quantidade de recursos disponíveis. Já os valores das 
folgas 2 e 3 que permaneceram na base, referem-se às sobras dos recursos. Assim, em um comparativo de resolução com o método Solver, os valores obtidos foram os mesmos, no entanto, os valores das restrições foram apresentados de forma diferente.

Após a resolução do proposto problema por dois métodos distintos, é possível afirmar que ambos alcançaram a mesma resposta. $\mathrm{O}$ diferencial dos métodos está no seu processo de resolução. O Solver, apresenta-se como o método mais simples e direto para a resolução do problema, visto que não é necessário o uso de nenhuma função e sucessivas iterações com cálculos, no entanto, a parametrização torna-se uma tarefa complexa para usuários iniciantes e, esse processo se torna dependente do uso de planilhas eletrônicas para funcionamento do suplemento.

A resolução do problema pelo método tabular, assim como o Solver apresenta vantagens e desvantagens. Por este método se basear em cálculos matemáticos, o uso de planilhas eletrônicas não é obrigatório, permitindo ao responsável pela resolução, solucionar manualmente o problema, o que torna-se uma vantagem na escassez de recursos computacionais. A não dependência de nenhum suplemento de qualquer software se apresenta como vantagem, além de disso, este método apresenta resultados após cada iteração, facilitando a compreensão durante a execução do processo. Todavia, sua desvantagem está ligada ao constante uso de funções matemáticas e sucessivos cálculos, e ainda em determinados casos, o grande número de iterações realizadas.

Destarte, ambos os métodos apresentam eficácia, porém, o Solver, mostra-se um método com menor nível de complexidade, pois, possui uma tela intuitiva para sua parametrização. Vale ressaltar que, ambos chegaram ao mesmo lugar, logo, quanto a sua aplicabilidade de cada método, deve ser analisada de acordo com a necessidade da proposta.

\section{CONSIDERAÇÕES FINAIS}

Se considerada as vantagens e desvantagens de cada um dos métodos utilizados, a problemática deste trabalho foi respondida ao ser definido que a resolução do problema de programação linear pelo método Solver, apresenta-se menos complexa, e ainda assim é mais eficaz que pelo método tabular, pois, abstrai a utilização de várias funções e cálculos.

Ambos objetivos desta temática foram cumpridos a partir da realização das revisões bibliográficas, modelagem e resolução do problema de Programação Linear além da comparação dos métodos utilizados na resolução do problema, almejando apontar qual apresenta menor complexidade.

Entretanto, mesmo que após a comparação realizada nessa temática aponte que o Solver apresenta-se o menos complexo, o método tabular, torna-se muito útil quanto há a necessidade de saber o valor de todas as variáveis a cada iteração do problema, apontando em qual parte da região de solução as variáveis de decisão estão percorrendo.

Conclui-se que um dos grandes diferenciais entre os dois métodos discutidos na pesquisa, é que o Solver apresenta maior velocidade de resolução do problema, uma vez que em uma pequena faixa de tempo e baixa complexidade, é possível realizar a parametrização da variáveis e a solução do problema, visto que a parte mais demorada de todo processo, é a construção do modelo matemático.

Persp. Online: exatas \& eng., Campos dos Goytacazes, 24 (09) 19-33 - 2019 
Sugere-se em trabalhos futuros a resolução de problemas de Progamação Linear reais, para que o resultado obtido, possa contribuir para a tomada de decisão. A partir desta resolução, apresentar ferramentas e/ou softwares para resolução dos problemas de Programação Linear.

\section{8 - REFERÊNCIAS}

ANDRADE, Eduardo Leopoldino de. (2004) Introdução a Pesquisa Operacional: Métodos e Modelos para Análise de Decisões. 4. ed. Rio de Janeiro: Ltc,. 204 p.

CAIXETA-FILHO, José Vicente (2004) Pesquisa Operacional: Técnicas de Otimização Aplicadas a Sistemas Agroindustriais. 2. ed. São Paulo: Atlas, 169 p.

Dantzig, G. B. (1998). Linear programming and extensions. Princeton university press.

GIL, Antonio Carlos. (2008) Métodos e Técnicas de Pesquisa Social. 6. Ed. São Paulo: Atlas, 200p.

HILLIER, Frederick S.; LIEBERMAN, Gerald J.. Introduction to operations research. 7. ed. New York: Mcgraw-hill, 2001. 1214 p. Disponível em: <https://notendur.hi.is/ kth93/3.20.pdf $>$. Acesso em: 23 ago. 2015.

LOESCH, Cláudio; HEIN, Nelson (2009) Pesquisa Operacional: Fundamentos e Modelos. São Paulo: Saraiva, 248 p.

LONGARAY, André Andrade (2013) Introdução a Pesquisa Operacional. São Paulo: Saraiva, 212 p.

PASSOS, Eduardo José Pedreira Franco dos (2008) Programação Linear: Como Instrumento da Pesquisa Operacional. São Paulo: Atlas, 451 p.

PRADO, Darci (2007) Programação Linear. 5. ed. Nova Lima: Indg Tecs, 238 p. (Pesquisa Operacional vol.1).

SILVA, Ermes Medeiros da et al. (2010) Pesquisa Operacional: Para os cursos de Administração e Engenharia. 4. ed. São Paulo: Atlas, 186 p.

Sociedade Brasileira de Pesquisa Operacional. Disponível em: $<$ http://www.sobrapo.org.br/o_que_e_po.php>. Acesso em 26 jun. 2015. 